\title{
Analysis of the spread of COVID-19 in Morocco based on a SEIR epidemic model
}

\author{
Moulay Rchid Sidi Ammia,*, Mostafa Tahiri ${ }^{\mathrm{b}}$ \\ ${ }^{a}$ Department of Mathematics, AMNEA Group, Faculty of Sciences and Techniques, \\ Moulay Ismail University of Meknes, B. P. 509, Errachidia, Morocco. \\ ${ }^{b}$ Department of Mathematics, AMNEA Group, Faculty of Sciences and Techniques, \\ Moulay Ismail University of Meknes, B. P. 509, Errachidia, Morocco.
}

\begin{abstract}
Fractional calculus has been widely used in mathematical modeling of evolutionary systems with memory effect on dynamics. In order to illustrate the efficiency of this non-integer order calculus, we employ SEIR models to model the dynamics, with and without memory, of the spread of Covid-19 in Morocco country.
\end{abstract}

Keywords: Mathematical modeling of COVID-19 pandemic, Numerical simulations, Basic reproduction number, Morocco case study.

2010 MSC: 34A08, 34K28.

\section{Introduction}

Infectious diseases have always been and continue to be of great concern to humanity since the beginning of its history. Infectious diseases are caused by pathogenic microorganisms, such as bacteria, viruses, parasites or fungi. Diseases can spread, directly or indirectly, from person to person or from animals to humans. The World Health Organization (WHO) reports that: Infectious diseases are responsible for a quarter to a third of all deaths worldwide each year. The vast majority occurring in low-income countries. Even when infections don't kill, they reduce the quality of life for hundreds of millions of people and stunt economic development. Population growth and spread, global climate change, and the emergence and re-emergence of new and deadly forms of infectious disease have increased the search for robust and effective ways to guide disease interventions. Over the past two decades, many epidemics have spread, including the SARS virus (2002) which killed 800 people around the world. In 2003, bird flu killed around 400 people. Then new types of flu appeared, such as the corona virus in the Middle East. In 2010, the WHO announced that 18,000 people had died from swine flu (H1N1). Other epidemics are also common, perhaps the most dangerous of which is the Ebola virus, which has spread to a number of african countries, and as a result, around 8,200 people have died from the virus. On December 31, 2019, the first suspected case was reported to the $\mathrm{WHO}$, as an emerging corona-virus, known as (COVID-19). It is the

\footnotetext{
${ }^{*}$ Corresponding author

Email addresses: rachidsidiammi@yahoo.fr (Moulay Rchid Sidi Ammi ), my .mustafa.tahiri@gmail . com (Mostafa Tahiri) doi:10.31559/glm2021.10.2.8
} 
most dangerous virus in history, because of its ability to spread rapidly in addition to its ability to mutate. More than hundreds of thousands of people died [12], so it crippled the worldwide economy. In many sciences, it is possible to conduct experiments to obtain information and test hypotheses. Experiments with the spread of infectious diseases in human populations are often impossible, immoral and costly. In most cases, the data is incomplete due to underreporting. This lack of reliable data makes it difficult to accurately estimate parameters, so that it may only be possible to estimate a range of values for certain parameters. Since repeatable experiments and precise data are generally not available in epidemiology, mathematical models and numerical simulations can be used, firstly, to perform the theoretical experiments needed for a variety of parameter values [2] and secondly to answer to important questions such as:

- Will there be an epidemic? If yes,

- How long will this last?

- How many people will be infected?

- Can the disease be eradicated by some control (isolation, quarantine, vaccination and treatment)?

The construction of a model aims, first of all, to understand the phenomenon studied. concerning modeling, the first thing to do, when faced with a real phenomenon, is to identify, label the independent and dependent variables and make sufficiently simplifying hypotheses, so that the case becomes mathematically treatable. The knowledge of the processes which govern the model and our mathematical knowledge are a precious help, which is useful for writing the equations connecting the various variables between them. The second step, in the modeling, consists in implementing our mathematical knowledge on the model, in order to derive theoretical results. Then, we put these results back into the real context, to draw conclusions under the form of explanations or predictions. The final step consists in verifying the predictions by comparing them with new real data. If the predictions do not match reality well, the model should be refined or formulated and the cycle started over. However, a mathematical model is not an ideal representation.of the real situation, but an approximate picture of the phenomenon studied. A good model is one that simplifies reality sufficiently to provide valid and useful conclusions. After the modeling process, one proceeds to the resolution of the obtained system. Here, the first step is to find the equilibrium points or fixed points of the system. Once these points have been obtained, one wonders about necessary and sufficient conditions of their stability. This is requires the study of the behavior of the system around these points. In general, we can conclude that epidemic modeling has three main purposes. Mathematical structure is the first essential part of understanding the spread of the disease. The second objective is to predict the future course of the epidemic. The third is to understand how we can control the spread of the epidemic.

The outline of the paper is as follows. Some basic results of fractional calculus are given in the second Section. Section 3 is dedicated to motivation for using fractional calculus by presenting two examples. Section 4 is devoted to an epidemic model of the SEIR type. To determine the robustness of model predictions to parameter values, sensitivity analysis is discussed in Section 5. Further, some numerical simulations are performed to compare the results of our model with the real data obtained from worldometers.

\section{Basic results of fractional calculus}

Definition 2.1. The Riemann-Liouville fractional integral of order $\alpha>0$ of a function $f \in L^{1}\left(\mathbb{R}^{+}\right)$is given by

$$
J^{\alpha} f(x)=\frac{1}{\Gamma(\alpha)} \int_{0}^{x}(x-t)^{\alpha-1} f(t) d t
$$

Here $\Gamma(n)=\int_{0}^{\infty} t^{n-1} e^{-t} d t$ 
Definition 2.2. Let $\alpha>0, n=[\alpha]+1, n-1<\alpha \leqslant n$, where $[\alpha]$ denotes the integer part of number $\alpha$. The Caputo fractional derivative of order $\alpha$ for a function $f \in \mathrm{C}^{\mathrm{n}+1}([a,+\infty), \mathrm{R})$ is defined by

$$
D^{\alpha} f(u)=J^{n-\alpha} D^{n} f(u)=\frac{1}{\Gamma(n-\alpha)} \int_{a}^{u} \frac{f^{(n)}(s)}{(t-s)^{\alpha+1-n}} d s, u>0 .
$$

\section{Motivations for using fractional calculus}

Fractional calculus adds information to classical calculus with a more precise description of certain natural phenomena. It can be applied in several fields of knowledge such as mechanics, viscoelasticity, bioengineering and control theory [3,4]. In this section, we present two motivations for using fractional calculus: the Tautochrone problem and the effect of memory in dynamical systems.

\subsection{Tautochrone problem}

The tautochronous (or isochronic curve) problem is finding a curve s for which an object spends the same time sliding through the curve for any starting point $y_{0}$ to 0 . The path is considered to be frictionless and under uniform gravity. In 1823, Abel solved this problem using fractional calculus [1]. The equation that describes the object's descent time is given by

$$
\tau=\frac{1}{\sqrt{2 g}} \int_{0}^{y_{0}}\left(y-y_{0}\right)^{-\frac{1}{2}}\left(\frac{d s}{d y}\right) d y
$$

where $g$ is the gravity, $y(t)$ is the height of the object at time $t, y_{0}$ is the initial height at which the object was launched, and $s$ is the curve desired given according to $y$. The curve $s$ can be found using classical calculus by applying the Laplace transform and the convolution theorem. Abel obtained the same solution using fractional calculus, in particular, he observed that except for the multiplication by $\frac{1}{\Gamma\left(\frac{1}{2}\right)}$, the equation (3.1) corresponds to the fractional integral of order $\frac{1}{2}$ of the function $s^{\prime}(y)$. Thus, one can easily obtain the desired solution if the fractional derivative of the constant $\tau$ is known.

\subsection{Memory effect: traditional approach}

Fractional calculus is a great tool that can be employed to describe real-life phenomena with socalled memory effect. A classic model of autonomous ordinary differential equations has no memory, because their solution does not depend on the previous instant. For instance, if $f\left(t ; x_{0}\right)$ is a solution of an autonomous first-order ordinary differential equation with an initial condition $x_{0}$ at $t=0$, then we have the flow property $f\left(t+s ; x_{0}\right)=f\left(t ; f\left(s ; x_{0}\right)\right)$, which means that the solution does not change by considering $f\left(s ; x_{0}\right)$ as initial condition since $f\left(s ; x_{0}\right)$ belongs to the solution. Thus, given an initial value, the solution is uniquely determined for any point of domain. In general, this assertion is not true for fractional differential equations. One way to introduce the memory effect into a mathematical model is to change the order of the derivative of a classical model so that it is non-integer [6]. Let $f$ be a real function defined in $[0, t], t_{1}, t_{2} \in[0, t]$ are such that $0<t_{1}<t_{2}$, and $H=\left(J^{\alpha} f\right)\left(t_{2}\right)-\left(J^{\alpha} f\right)\left(t_{1}\right)$ for $\alpha \in R_{+}$. From equalities below, one can observe that the value of $H$ depends on the entire range of $f$ over $\left[0, t_{2}\right]$ if $\alpha \neq 1$, whereas $H$ depends only on the range of $f$ over $\left[t_{1}, t_{2}\right]$ if $\alpha=1$ :

$$
\begin{aligned}
H & =\left(J^{\alpha} f\right)\left(t_{2}\right)-\left(J^{\alpha} f\right)\left(t_{1}\right) \\
& =\frac{1}{\Gamma(\alpha)}\left[\int_{0}^{t_{2}}\left(t_{2}-s\right)^{\alpha-1} f(s) d s-\int_{0}^{t_{1}}\left(t_{1}-s\right)^{\alpha-1} f(s) d s\right] \\
& =\frac{1}{\Gamma(\alpha)}\left[\int_{t_{1}}^{t_{2}}\left(t_{2}-s\right)^{\alpha-1} f(s) d s+\int_{0}^{t_{1}}\left[\left(t_{2}-s\right)^{\alpha-1}-\left(t_{1}-s\right)^{\alpha-1}\right] f(s) d s\right]
\end{aligned}
$$


Note that if $\alpha=1$, then the second integral is canceled:

$$
H=\frac{1}{\Gamma(\alpha)} \int_{t_{1}}^{t_{2}}\left(t_{2}-s\right)^{\alpha-1} f(s) d s=\int_{t_{1}}^{t_{2}} f(s) d s .
$$

In contrast, the second integral remains if $\alpha \neq 1$. From (3.2) we can see that $H$ depends on what happens in $\left[0, t_{1}\right]$ and $\left[t_{1}, t_{2}\right]$. Thus, for $\alpha \neq 1, H$ depends on the entire range of $f$ over $\left[0, t_{2}\right]$. Therefore, the fractional integral in the interval $\left[t_{1}, t_{2}\right]$ is not uniquely determined; it depends on what happened before $t_{1}$ which characterizes the memory effect in the process.

\section{Numerical Simulations}

In this section we analyze the spread of Covid-19 in Morocco using an epidemic model of the SEIR type. In [10], authors pointed out that the effect memory should ideally be considered in the analysis of the epidemiological behavior of diseases due to actions caused by the protective instinct and immunity, which are influenced by the so-called immune memory of the body. Since we desire to incorporate the memory effect, we use the fractional version of the SEIR model.

\subsection{Proposed COVID-19 model}

We propose a new epidemiological compartment fractional model taking into account the effect of the lockdown of susceptible individuals. The total population of size $\mathrm{N}$ is subdivided into the following epidemiological classes:

- Susceptible class $S(t)$ : the number of uninfected individuals at the time $t$.

- Exposed class $E(t)$ : the number of infected individuals at the time $t$ but still in incubation period (without clinical symptoms).

- Infected class $I(t)$ : the number of infected individuals at the time $t$ (with obvious clinical symptoms).

- Recovered class $R(t)$ : the number of recovered individuals at the time $t$.

- Insusceptible class $\mathrm{P}(\mathrm{t})$ : the number of susceptible individuals who are not exposed to the external environment at the time $t$.

Our fractional model takes the following form:

$$
\left\{\begin{array}{l}
{ }_{0}^{C} D_{t}^{\alpha} S(t)=\Lambda-\beta_{1}(1-\delta)(1-\lambda) S(t) I(t)-\beta_{2} S(t) E(t)-\lambda S(t)-\mu S(t), \\
{ }_{0}^{C} D_{t}^{\alpha} E(t)=\beta_{1}(1-\delta)(1-\lambda) S(t) I(t)+\beta_{2} S(t) E(t)-\xi E(t)-\mu E(t), \\
{ }_{0}^{C} D_{t}^{\alpha} I(t)=\xi E(t)-(\rho \delta+d \delta) I(t)-\mu I(t), \\
{ }_{0}^{C} D_{t}^{\alpha} R(t)=\rho \delta I(t)-\mu R(t), \\
{ }_{0}^{C} D_{t}^{\alpha} P(t)=\lambda S(t)-\mu P(t),
\end{array}\right.
$$

where the fractional derivative is considered in the sense of Caputo. For biological reasons, we consider system (4.1) with the following initial conditions:

$$
S(0) \geqslant 0, \quad E(0) \geqslant 0, \quad I(0) \geqslant 0, \quad R(0) \geqslant 0, \quad P(0) \geqslant 0 .
$$

Different parameters intervening in the model are presented in the Table 1.

The number of death due to the disease at each instant of time is given by

$$
\mathrm{D}(\mathrm{t}):=\mathrm{d} \delta \mathrm{I}(\mathrm{t}) \text {. }
$$


Table 1: Values of the model parameters corresponding to the situation of Morocco.

\begin{tabular}{|l|l|l|l|}
\hline Name & Description & Value & Unit \\
\hline$\Lambda$ & Birth density of susceptible & $6.00 \times 10^{5}$ & dimensionless \\
$\beta_{1}$ & Transmission coefficient due to infected individuals & 0.87 & day $^{-1}$ \\
$\delta$ & Isolation rate of infected & 0.17 & day $^{-1}$ \\
$\lambda$ & Protection rate of susceptible & 0.04 & day $^{-1}$ \\
$\beta_{2}$ & Transmission coefficient due to exposed individuals & 0.90 & day $^{-1}$ \\
$\mu$ & Natural death rate & $1.00 \times 10^{-4}$ & day $^{-1}$ \\
$\xi$ & Rate at which exposed people become infected & 0.74 & day $^{-1}$ \\
$\rho$ & Recovery rate coefficient & 0.81 & day $^{-1}$ \\
d & Death rate coefficient due to infected & $16.00 \times 10^{-3}$ & day $^{-1}$ \\
\hline
\end{tabular}

\subsection{Basic reproduction rate}

In this section, we discuss a fundamental idea in epidemiology which is the existence of a threshold value according to certain parameters depending on the disease and allowing to predict if a disease will become an epidemic or not. The value

$$
R_{0}=\frac{\xi \beta_{1} \Lambda(1-\delta)(1-\lambda)}{(\xi+\mu)(\lambda+\mu)(\rho \delta+\mu+d \delta)}+\frac{\beta_{2} \Lambda}{(\xi+\mu)(\lambda+\mu)}
$$

is known as the basic reproduction rate, that is the average number of new cases of infection, caused by an infected individual during the period of infectivity, in a population entirely made up of susceptible individuals [11]. In our case, we obtain $R_{0}=1.2254$. This epidemiologically means that the disease will persist in the population.

\section{Sensitivity Analysis}

The sensitivity analysis for the basic reproduction number (4.2) tells how each parameter is important to disease transmission. This information is crucial not only for experimental design, but also to data assimilation and reduction of complex models [8]. Sensitivity analysis is commonly used to determine the robustness of model predictions to parameter values, since there are usually errors in collected data and presumed parameter values. It is used to discover parameters that have a high impact on the threshold $R_{0}$ and should be targeted by intervention strategies. More accurately, sensitivity indices permit to measure the relative change in a variable when a parameter changes. For that purpose, we use the normalized forward sensitivity index of a variable with respect to a given parameter, which is defined as the ratio of the relative change in the variable to the relative change in the parameter. If such variable is differentiable with respect to the parameter, then the sensitivity index is defined as follows.

Definition 5.1 (See $[5,9]$ ). The normalized forward sensitivity index of $R_{0}$, which is differentiable with respect to a given parameter $\theta$, is defined by

$$
\Upsilon_{\theta}^{R_{0}}=\frac{\partial R_{0}}{\partial \theta} \frac{\theta}{R_{0}}
$$

Note that the sensitivity index may depend on several parameters of the system, but also can be constant, independent of any parameter. For example, $\Upsilon_{\theta}^{R_{0}}= \pm 1$ means that increasing (decreasing) $\theta$ by a given percentage increases (decreases) always $R_{0}$ by that same percentage. The estimation of a sensitive parameter should be carefully done, since a small perturbation in such parameter leads to relevant quantitative changes. On the other hand, the estimation of a parameter with a rather small value for the sensitivity index does not require as much attention to estimate, because a small perturbation in that parameter leads to small changes. The results of this analysis are presented in the Table 2. 
Table 2: Sensitivity of $R_{0}$ evaluated for the parameter values given in Table 1.

\begin{tabular}{|c|c|}
\hline Parameter & Sensitivity index \\
\hline$\wedge$ & 0.990 \\
$\beta_{1}$ & 0.600 \\
$\delta$ & -0.120 \\
$\lambda$ & -0.920 \\
$\beta_{2}$ & 0.140 \\
$\mu$ & -0.006 \\
$\xi$ & -0.136 \\
$\rho$ & -0.590 \\
$d$ & -0.012 \\
\hline
\end{tabular}

\subsection{Results}

In the following discussion, we perform numerical simulations to compare the results of our model with the real data obtained from worldometers [12]. The system (4.1) is numerically integrated by using the fractional Euler's method which can be seen as a generalization of the classical Euler's method for the numerical solution of ordinary differential equations [7]. The accuracy of the approximation depends on the step size of discretization. It is worthwhile to mention that Morocco has a population of about 34 million. As for the initial conditions, the following values have been fixed: $S(0)=33993018, E(0)=417$, $\mathrm{I}(0)=3460, \mathrm{R}(0)=3038, \mathrm{D}(0)=67$. We can estimate some parameters' values of Morocco from November $1^{\text {st }}$ to November $13^{\text {th }}$ (see Table 1). In the Figures 1 and 2, we remark the increase in the number of infectious cases and death cases. We remark also the fitting effect of the fractional order system (4.1) notably for $(\alpha=0.5)$ is better than that of the integer order system. It comes down to the memory effect which represents precautions taken by susceptible individuals.

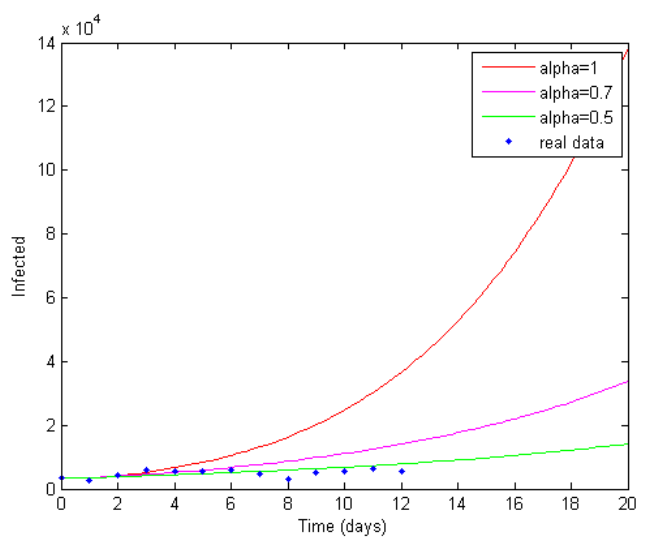

Figure 1: Number of infected cases per day, by using a Matlab code.

\section{Conclusion}

In this work, we showed that our model can be adjusted to real data. Then, we have obtained a good prediction of the evolution of the disease for $\alpha=0.5$. We hope our work motivates new researches to give significant improvements, especially the suitable choice of the incidence function which models the transmissibility from asymptomatic individuals. 


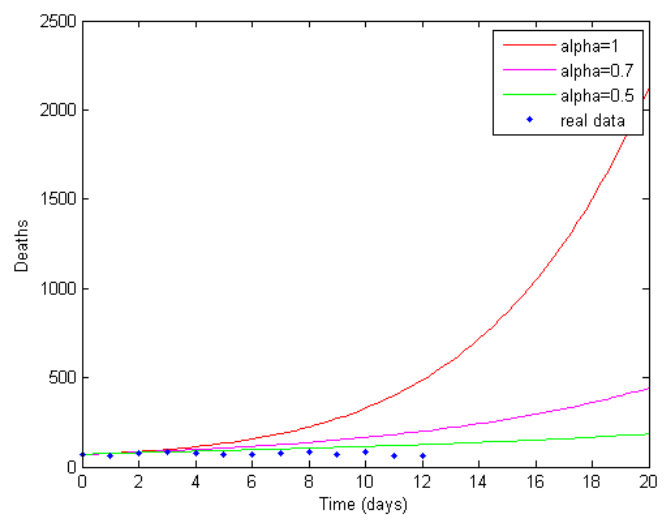

Figure 2: Number of death cases per day, by using a Matlab code.

\section{References}

[1] N. Abel. Solution de quelques problèmes à l'aide d'intégrales définies, Euvres, 1 (1881), 11-27. 3.1

[2] L. Acedo, G. González-Parra, and A. J. Arenas. An exact global solution for the classical SIRS epidemic model, Nonlinear Analysis: Real World Applications, 11 (2010), no. 3, 1819-1825. 1

[3] R. P. Agarwal, D. Baleanu, J. J. Nieto, D. F. Torres, and Y. Zhou. A survey on fuzzy fractional differential and optimal control nonlocal evolution equations, Journal of Computational and Applied Mathematics, 339 (2018), 3-29. 3

[4] R. Almeida, and D. Tavares, and D. F. M. Torres. The variable-order fractional calculus of variations, Springer, 2019. 3

[5] N. Chitnis, J. M. Hyman and J. M. Cushing. Determining important parameters in the spread of malaria through the sensitivity analysis of a mathematical model, Bulletin of Mathematical Biology, 70 (2008), 1272-1296. doi:10.1007/s11538008-9299-0. 5.1

[6] K. Diethelm. The analysis of fractional differential equations: An application-oriented exposition using differential operators of Caputo type, Springer Science \& Business Media, 2010. 3.2

[7] Z. Odibat and S. Momani. An algorithm for the numerical solution of differential equations of fractional order, J. Appl. Math. Inform., (2008), 26:15-27. 5.1

[8] D. R. Powell, J. Fair, R. J. LeClaire, L. M. Moore and D. Thompson. Sensitivity analysis of an infectious disease model, Proceedings of the International System Dynamics Conference; Boston, M., Ed., 2005. 5

[9] H. S. Rodrigues, M. T. T. Monteiro and D. F. M. Torres. Sensitivity analysis in a dengue epidemiological model, Conference Papers in Mathematics; Hindawi., Ed., 2013 (2013), Art. ID 721406. doi:10.1155/2013/721406. arXiv:1307.0202 5.1

[10] M. R. Sidi Ammi, M. Tahiri and D. F. M. Torres, Global stability of a Caputo fractional SIRS model with general incidence rate, Mathematics in Computer Science, (2020). 4

[11] P. Van den Driessche and J Watmough. Reproduction numbers and sub-threshold endemic equilibria for compartmental models of disease transmission, Mathematical biosciences, 180 (2002), 29-48. 4.2

[12] https://www.worldometers.info/coronavirus/country/morocco/ 1, 5.1 